\title{
Application of Vibratometry in Evaluation of Frequency Detection and Signal Intensity in Patients with Unilateral Cochlear Implants - Preliminary Results
}

\author{
A. Sekula ${ }^{a, c}$, A. Pruszewicz ${ }^{a}$, O. Stieler ${ }^{b, c, *}$, R. Gibasiewicz $^{b}$, M. Karlik $^{a}$ \\ AND D. KOMAR ${ }^{c}$ \\ ${ }^{a}$ Department of Phoniatrics and Audiology, Poznań University of Medical Sciences, Poznań, Poland \\ ${ }^{b}$ Department of Otolaryngology, Poznań University of Medical Sciences \\ S. Przybyszewskiego 49, 60-355 Poznań, Poland \\ ${ }^{c}$ Hearing Therapy Center KIND, Poznań, Poland
}

\begin{abstract}
Vibratometry is a unique audiological test used in patients with bilateral profound sensori-neural hearing loss. An acoustic stimulus is presented using the bone conduction. The study comprised 15 patients with bilateral profound hearing loss with unilaterally implanted cochlear implant. Vibration detection thresholds were noted. Measurements were done at the implanted side with speech processor either switched on or switched off, as well as at the contralateral side with speech processor switched on. All the patients have perceived stimulus used for testing. The average detection thresholds of vibration stimulus and average thresholds in bone conduction using tonal stimulus have shown better perception of vibration delivered by bone conduction than tonal stimulus. The average detection threshold of vibration stimulus in the implanted ear showed significantly better results in case of speech processor switched on. Patients perceived high frequency stimuli during vibratometry testing despite of no reaction to tonal stimulation during bone conduction test. Vibration could be a supplementary stimulation in patients with bilateral perceptive profound hearing loss.
\end{abstract}

PACS: 43.66. $+\mathrm{y}, 43.60 .-\mathrm{c}, 34.50 . \mathrm{Ez}$

\section{Introduction}

Vibratometry is a unique audiological test used in patients with bilateral profound sensori-neural hearing loss. There are only a few reports describing studies of vibration stimulus' perception in audible frequency range in patients with hearing loss $[1,2]$. An acoustic stimulus is presented using the bone conduction. A comparison of detection threshold of this vibration stimulus to the hearing threshold for bone conduction (in a pure tone audiometry using the frequency range of $125 \mathrm{~Hz}$ to $4000 \mathrm{~Hz}$ ) shows a significantly better perception of vibration stimulus than the perception of tonal stimulus in patients with profound hearing loss [3].

There is a phenomenon observed during vibratometry measurement that the deaf patient or the patient with residual hearing can perceive presented stimulus as an audible one. There is still a question to answer, how a patient with hearing loss can perceive this sound using neural afferent way? There are some hypotheses which take into consideration the following neural pathways:

* corresponding author; e-mail: osaku@umed.poznan.pl auditory pathway of 8th cranial nerve, non-specific auditory pathway (anastomoses with facial nerve), non-specific pathway by stimulation the vestibular organ, deep sensation pathway (prioprioceptive pathway) [4-6].

The vibratometry test is used in qualification procedure for cochlear implantation (CI) as a prognostic factor just to predict the future efficacy of implanted device $[1,2]$. The patient qualified for cochlear implant surgery should have at least profound bilateral hearing loss. Disadvantages of unilaterally implanted devices combined with a head shadow effect are commonly known [7, 8]. To avoid this head shadow effect the following solutions can be used: (a) bilateral implantation (due to the high cost it is a procedure not commonly used in Poland), (b) bimodal stimulation, which means usage of CI on the one side and hearing aid (HA) on contralateral side (patients with a small acoustic gain from the hearing aid do not accept bimodal stimulation), (c) similar to contralateral routing of signal (CROS) signal transfer system theoretical solution.

Based on the previously published results [1, 2] which emphasized the better perception of vibration (delivered by bone conduction) than tonal stimulus in deaf patients 
we decided to test unilaterally implanted patients using vibratometry.

The aim of our preliminary study was to compare a perception of sound during vibratometry test in the implanted ear with the contralateral one (without implant) and to refer the results to the hypothetical afferent pathways, which allow a perception of sound in deaf patients with cochlear implants.

\section{Material}

The study comprised 15 patients with bilateral profound hearing loss, aged 15-62 years (mean: 35.6 years), including 10 females and 5 males, who showed the possibility of vibration reception. Patients without this reception could not be included into the study. All the patients have been implanted unilaterally with cochlear implant and they have been experienced recipients, using the device for 0.5-10 years (mean: 3.3 year).

\section{Methods}

In all the patients vibratometry test was performed. A stimulus presented was a filtered noise and was delivered to bone vibrator placed on the mastoid process of the temporal bone. Frequencies of stimuli were compatible with the frequencies used in audiometric tests. Vibration detection thresholds were noted.

At the beginning a pure tone audiometry test with air and bone conduction thresholds was done. Then, hearing thresholds in cochlear implants using free field technique were evaluated. Vibratometry measurements were done at the implanted side with speech processor either switched on or switched off. Next, stimulation of the contralateral side was done with speech processor (at the implanted side) switched on.

The ability of patients to differentiate $5 \mathrm{~dB}$ loudness increments for 2 tested frequencies ( 1 and $2 \mathrm{kHz}$ ) was also observed.

\section{Results}

All the patients have perceived presented vibratory stimulus. Figure 1 presents the number of patients perceiving sounds for every tested frequency. Four bars shows appropriately: (1) bone conduction in the implanted ear (BC), (2) vibratometry in the implanted ear without speech processor (VIB), (3) vibratometry in the implanted ear with the speech processor $(\mathrm{VIBipsi}+\mathrm{CI}$ ), (4) vibratometry in the contralateral ear with the speech processor (VIBcontra $+\mathrm{CI}$ ). It can be observed that stimuli in the frequency range of $2-6 \mathrm{kHz}$ were perceived by the patients only during vibratometry test (bars VIP, VIPipsi + CI, VIPcontra $+\mathrm{CI}$ ), while the bone conduction was not noticed. In the frequency range of 4-6 kHz only bars VIBipsi + CI and VIPcontra + CI are present, showing perception of vibration stimulus only when speech processor was switched on. For all tested frequencies higher numbers of patients were found when speech processor was switched on (bars VIBipsi+CI, VIBcontra $+\mathrm{CI}$ ) indicating influence of active speech processor on vibration perception.

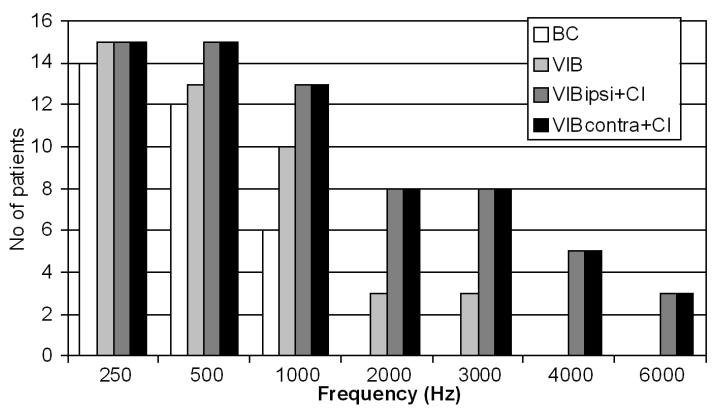

Fig. 1. Number of patients perceiving tested frequencies for different stimulation modes.

The average detection thresholds of vibration stimuli as well as average bone conduction thresholds (using tonal stimuli) confirmed earlier published results indicated better perception of vibration delivered by bone conduction than perception of tonal stimulus. Figure 2 shows lower detection thresholds for vibration stimulus and wider stimulation frequency range (till $3 \mathrm{kHz}$ ).

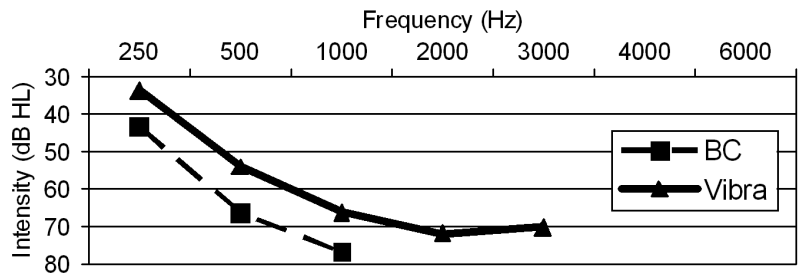

Fig. 2. The average detection thresholds of bone conduction (BC) and average vibratometry thresholds (VIB).

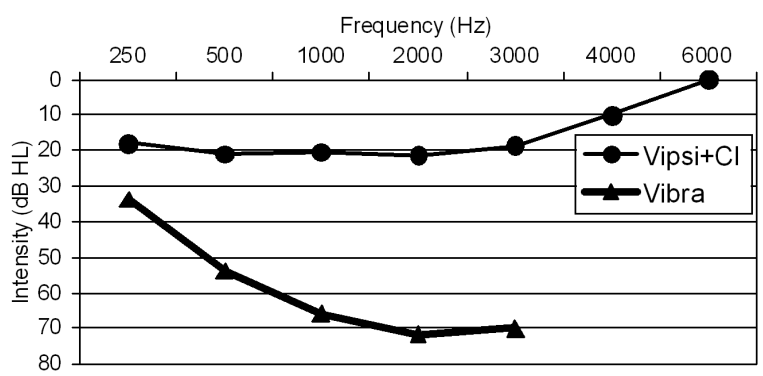

Fig. 3. The average detection threshold of vibration in the implanted ear in case of speech processor switched on (VIBipsi $+\mathrm{CI}$ ) and without the speech processor (VIB).

The average detection thresholds of vibration stimulus measured at the implanted side compared to the thresholds noticed when speech processor was active are pre- 


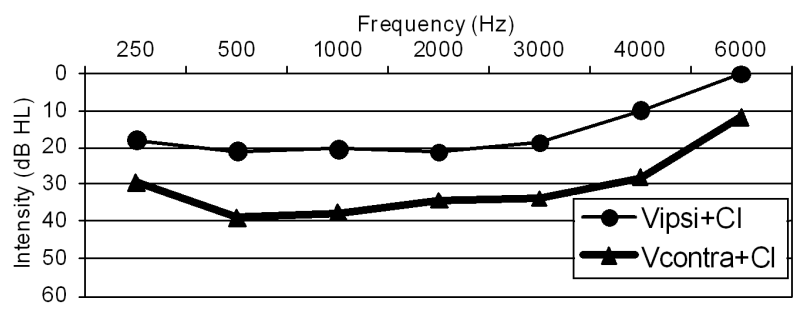

Fig. 4. The average detection thresholds of vibrations with speech processor switched on in the implanted ear (VIBipsi $+\mathrm{CI}$ ) and in contralateral ear (VIBcontra $+\mathrm{CI}$ ).

sented in Fig. 3. We observed significantly better thresholds and wider frequency range (till $6 \mathrm{kHz}$ ) in case of speech processor switched on.

Figure 4 shows the average detection thresholds of vibration stimulus in the implanted ear comparing contralateral ear with the speech processor switched on in both measurements. Better results can be clearly seen in the ear with cochlear implant (VIBipsi $+\mathrm{CI}$ ).

The ability to differentiate $5 \mathrm{~dB}$ loudness increments for 2 tested frequencies ( 1 and $2 \mathrm{kHz}$ ) was demonstrated by all the patients.

\section{Discussion}

Perceiving a stimulus used in vibratometry gives us an important information about the possibility of stimulation of auditory system in patients with hearing loss. Lower detection vibration thresholds observed during our preliminary study, comparing to perception of tonal signals transmitted by bone conduction, shows a new possibility of receiving information by these hearing impaired patients. A quite new observation is a contralaterally registered perception of signals used during vibratometry tests as an audible signal in the implanted ear. An attenuation of the signal transmitted by this way to the microphone of speech processor does not exceed $20 \mathrm{~dB}$, so it could be used as a potential method to obtain directional hearing in deaf patients with unilaterally implanted cochlear implants and to overcome head shadow without using second implant.
Based on this preliminary report we plan to extend our research on the perception of vibration signal by the bone conduction in bilaterally deaf patients. Till now, no reports were noted on using acoustic vibration in the rehabilitation of cochlear implant recipients.

\section{Conclusions}

Our early results show better detection thresholds of vibration stimuli measured at the implanted side when speech processor was active. Detection thresholds of vibration with speech processor switched on were better in the ear with cochlear implant comparing contralateral ear. Patients perceived high frequency stimuli during vibratometry testing despite of no reaction to high frequency tonal stimulation during bone conduction test. Vibratometry could enable future evaluation of benefit from bimodal stimulation. Vibration could be a supplementary stimulation in patients with bilateral perceptive profound hearing loss.

\section{References}

[1] A. Pruszewicz, W. Szyfter, E. Szymiec, B. Bilińska, in: IIIrd Int. Congress on Cochlear Implant, Paris, 1995, abstract book, p. 105.

[2] A. Pruszewicz, P. Świdziński, A. Obrębowski, B. Bilińska, M. Karlik, in: 1st Int. Symp. "Rehabilitation in Otology", Poznań, 1998, abstract book, p. 31.

[3] P. Świdziński, A. Pruszewicz, in: Clinical Audiology, Outline, Eds. A. Pruszewicz, A. Obrębowski, Wydawnictwo Naukowe Uniwersytetu Medycznego, Poznań 2010, p. 210 (in Polish).

[4] R. Skórnicki, A. Obrębowski, Folia Morph. 25, 608 (1966).

[5] A. Obrębowski, R. Skórnicki, Folia Morph. 26, 186 (1967).

[6] R. Skórnicki, A. Obrębowski, Anatomischer Anzeiger 122, 149 (1968).

[7] D.E. Rose, J. Haymond, G.W. Facer, Otolaryngol. Head Neck Surg. 99, 42 (1988).

[8] A.B. King, M.C. Martin, Br. J. Audiol. 20, 19 (1986). 